\title{
Philosophiques
}

\section{Regard sur la philosophie de la technique en Allemagne - L'École de Francfort et la NGT : analogies et différences}

\section{Lazare Marcelin Poamé}

Volume 21, numéro 1, printemps 1994

URI : https://id.erudit.org/iderudit/027257ar

DOI : https://doi.org/10.7202/027257ar

Aller au sommaire du numéro

Éditeur(s)

Société de philosophie du Québec

ISSN

0316-2923 (imprimé)

1492-1391 (numérique)

Découvrir la revue

Citer cet article

Poamé, L. M. (1994). Regard sur la philosophie de la technique en Allemagne L'École de Francfort et la NGT : analogies et différences. Philosophiques, 21(1), 197-211. https://doi.org/10.7202/027257ar d'utilisation que vous pouvez consulter en ligne. 


\section{REGARD SUR LA PHILOSOPHIE DE LA IECHNIOUE EN ALLEMAGNE}

\section{L'École de Francfort et la NGT ${ }^{1}$ : analogies et différences}

\section{par Lazare Marcelin Poamé}

En 1984, Gilbert Hottois, dans Le signe et la technique déclarait : « La philosophie de la technique est une discipline encore fort jeune et mal assuree de son identité. Elle ne se distingue pas toujours nettement de la philosophie des sciences, de la philosophie politique (plus généralement de la philosophie sociale) : elle est, comme on le voit, tellement incertaine d'elle-même qu'elle oscille entre les extrêmes opposés ${ }^{2}$.

Ce propos non moins pertinent que révélateur draine des appréhensions qui gagnent de moins en moins du terrain dans la communauté philosophique occidentale notamment aux USA, en France ${ }^{3}$ et surtout en Allemagne. Il nous semble en tout cas difficile de soutenir aujourd'hui - et cela en regard de l'espace philosophique germanique - que la philosophie de la technique est une discipline mal assuree de son identité ...

Mais qu'en est-il exactement de cette discipline en Allemagne ${ }^{4}$ ? C'est la question à laquelle tente de répondre modestement la présente

I. NGT : formule abrégée de neue Generation der Technikphilosophen qui signifie nouvelle genération des philosophes de la technique.

2. Hottois (G.) : Le signe et la technique. La philosophie à l'épreuve de la technique, Paris, Aubier Montaigne, 1984, p. 17.

Cet ouvrage resté jusqu'à ce jour notre livre de chevet est dans l'espace philosophique francophone l'une des rares productions abordant de front les problèmes majeurs de la philosophie de la technique.

3. On connaît les efforts de F. Tinland, D. Cérézuelle, J.-Y. Goffi, et de J.-P Schneider à cet egard. L'attention particulière qu'ils portent à la philosophie de la technique permettra à cette discipline de trouver dans le système educatif français, voire francophone, la place qui doit être la sienne.

4. Signalons au passage que pendant notre long sejjour universitaire allemand, nous avons nous-même participé (et même activement) à des séminaires de 
étude. La tâche qui nous incombe ici se résume en une présentation du paysage germanique de la philosophie de la technique.

Pour l'accomplissement de cette tâche, (au moins) deux perspectives sont envisageables. On peut opter pour une approche historique de la question en restituant dans le détail les étapes de cette discipline dénommée Philosophie der Technik ou Technikphilosophie ou encore et en français « philosophie de la technique ». On peut également préférer l'approche conceptuelle en insistant beaucoup plus sur ce qui se loge dans le concept de Technikphilosophie que sur son évolution.

Irréductible à chacune de ces deux démarches, notre propos se veut une synthèse de celles-ci. Cette orientation commandée par un souci d'originalité sera pour l'essentiel une confrontation des thèses classiques de l'École de Francfort avec celles des philosophes connus en Allemagne sous la dénomination de neue Generation der Technikphilosophen. Mais la confrontation que nous nous proposons ici de conduire ne prend tout son sens que dans l'échiquier (topique) de la Technikphilosophie qui est du reste quasi inséparable de son histoire.

\section{Txcursus sur la phillosophie de la technique}

Tous les penseurs de la technique (Technikphilosophen) en Allemagne reconnaissent unanimement que la philosophie de la technique a une histoire, voire un état civil; ils s'accordent à admettre qu'elle a reçu son acte de baptême en 1877 avec la parution des Grundlinien einer Philosophie der Technik d'Ernst Kapp. La thèse fondamentale des Grundlinien ... se résume en une affirmation vigoureuse de l'analogie entre l'organique et le technique. Dans cette analogie, l'organique est posé comme archétype. Le point de départ de cette analogie inspirée d'Aristote, est la main considérée comme le modèle des instruments (Vorbild fur mechanische Werkzeuge). Poursuivant l'analogie, Kapp trouve dans la vue et l'ouie le modèle des instruments et appareils acoustiques. Aussi, perçoit-il dans les os (Knochen) la forme archétypale (Urbild) des pièces de construction (Konstruktionsteil) ${ }^{5}$.

A la suite de Kapp, Friedrich Dessauer publie un ouvrage dont le titre assez évocateur indique que la philosophie de la technique a quitté les Grundlinien (sa phase introductive) et est désormais entrée dans sa phase décisive. Il s'agit de Die Philosophie der Technik, un ouvrage paru en

« Philosophie der Technik » animés par l'Institut polytechnique et le Département de philosophie de l'Université de Francfort.

5. Ces vues initiées par E. Kapp seront reprises et systématisées par A. Gehlen le promoteur du concept de projection organique (Organprojektion). 
1927 qui définit la technique comme être procédant des Idées (Sein aus Ideen ou Realisierungvon Ideen). Chez Dessauer, les Idées ont une existence propre, un monde qui leur est propre (Reich der Ideen) et préexistent à l'objet technique qui vient à l'existence par nos mains. Avec cette conception de la technique, Dessauer donne visiblement dans la métaphysique et plus exactement dans une métaphysique d'inspiration platonicienne.

Cet horizon qui est celui de la métaphysique sera « repris » plus tard par Martin Heidegger sous la forme d'une «technologie ». Heidegger « technologue »! Surtout connu pour sa dénonciation de l'oubli de l'Être, Martin Heidegger paraît bien plus attentif à la réalité technique qu'on ne pense. Mais cette attention est liée à l'essentiel, c'est-à-dire l'essence de la technique. Cette essence qu'il fait résider dans l' Arraisonnement (das Gestell), est pour le philosophe ce qu'il y a de plus préoccupant dans la technique. C'est pourquoi, en affirmant que « la position fondamentale des Temps nouveaux est la position technique ${ }^{6} \gg$, il invite à voir dans la technique autre chose qu'une simple poiesis

Ce qu'il faut retenir de ce bref parcours que nous venons de retracer est que les perspectives initiées par les auteurs précités s'inscrivent dans un registre particulier de la philosophie de la technique dénommé traditionellement Technikphilosophie. S'inscrivent également dans ce registre, les thèses qui posent la technique comme :

- Science appliquée (Reuleaux. Bunge et dans une certaine mesure Rumpf).

- Objectivation du travail humain (Gehlen).

- Puissance autonome venant s'imposer aux hommes avec la force d'un destin aveugle (Schelsky).

- Système de moyens ambivalents (tête de Janus) ou ensemble d'instruments neutres (Jaspers, Sachsse, Tondl).

- Émancipation des entraves de la nature (Freyer).

- Système de moyens au service de la domination de la nature (Realtechnik de Gottl-Ottlilienfeld).

L'une des faiblesses de la traditionelle Technikphilosophie est de n'avoir pas été à la fois assez critique et pragmatique à l'égard de la technique. C'est en tout cas l'un des reproches que lui adressent les tenants

6. Heidegger, M. : Concepts fondamentaux, traduction Pascal David, Paris, Gallimard, 1985, P. 31. 
de la version « moderne ${ }^{7} \gg$ de la philosophie de la technique. Si la traditionnelle Technikphilosophie accuse des faiblesses, il faudra sans doute recourir à l'École de Francfort (réputée pour sa critique) et surtout à la NGT pour espérer combler ces déficiences.

\section{Il.l'Ćcole de Franclort et les nouveauu philosophes de la technique A. Contribution de l'École de Francfort à la philosophie de la technique}

Quatre grandes figures peuvent être ici invoquées à savoir Max Horkheimer, Theodor W. Adorno, Herbert Marcuse et Jürgen Habermas ${ }^{8}$.

Au sujet de Horkheimer, Friedrich Rapp écrit : « Pour ce qui est de la philosophie de la technique, l'œuvre de Horkheimer parue sous le titre: Zur Kritik der instrumentellen Vermunft, est capitale ${ }^{9} \gg$ Ce qu'il y a de significatif dans l'œuvre de Max Horkheimer nous paraît être entre autres le procès engagé contre la « raison instrumentale ». Ce qu'il appelle raison instrumentale, est la raison dénuée de tout contenu objectif, une raison en proie à la déchéance. Cette raison déchue, il l'appelle « raison subjective » et lui oppose la « raison objective ». Contrairement à cette dernière qui se veut fin en soi, autonome, la raison subjective, abandonnant quant à elle l'autonomie, « se conforme à n'importe quoi $»^{\mathrm{O}}$; elle se présente pour ainsi dire comme « une finalité sans fin qui, de ce fait, peut s'attacher à toutes les fins $\gg^{\mathrm{II}}$.

A en croire Horkheimer, cette raison dite subjective est incarnée dans la technique moderne, laquelle se révèle comme instrument de pouvoir non pas au service de l'homme contre la nature mais comme instrumentalisation de la nature et de l'homme. Et ce qui peut résulter de ce processus d'instrumentalisation, ce sont entre autres l'« éclipse

7. Cette version « modeme » a pris son essor à la fin des années soixante sous l'impulsion de la « NGT ».

8. Nonobstant la césure introduite au sein de cette Ecole par Habermas, nous nous emploierons à faire marcher de front les auteurs précités en faisant apparaître ce qui pour la circonstance les rassemble, à savoir la critique de la rationalité technique. Cette critique étant aujourd'hui devenue classique, nous ne nous y attarderons pas.

9. Rapp, F., Analytische Technikphilosophie, Munich, Editions Karl Albert, 1978, p. 22. Certes, mais on ne saurait pour autant négliger les autres écrits (tels Dialektik der Aufklärung, Gesellschaft im Übergang...) qui nous paraissent tout aussi significatifs que l'cuvre de 1947 .

Io. Horkheimer, M., Kritik zur instrumentellen Vernunft, Frankfurt, Fischer, 1967, p. 34.

II. Horkheimer / Adorno, Dialektik der Aufklärung, Amsterdam, Querido Verlag, 1955, p. I08-1og. 
de la raison », « le déclin de l'individu » et la perte du sens, corollaire de la réification du langage et de la pensée. Horkheimer rappelait à cet effet que « plus les idées sont devenues instrumentalisées [...] moins on a vu en elles des pensées dotées du sens qui leur appartient en propre. On les considère, poursuit-il, comme des choses, des machines. Et dans le gigantesque appareil de production de la société moderne. le langage a été réduit à n'être plus qu'un outil comme les autres. Toute phrase qui n'est pas équivalente à une opération de cet appareil semble aussi dépourvue de sens $\gg^{\mathbf{1 2}}$.

Cette raison subjective / instrumentale qui se prête à la liquidation du sens et de l'essence langagière de l'homme est également annihilation des différences. Posée dans l'arché de la civilisation technicienne, elle « confère à tout un air de ressemblance. Le film, la radio et les magazines constituent un système. Chaque secteur est uniformisé et tous le sont les uns par rapport aux autres $\gg^{13}$.

Ce passage est assez révélateur et mérite attention. Il indique clairement que les objets techniques sont solidaires les uns des autres; qu'ils ne sont pas une juxtaposition d'objets divers. Et c'est à juste titre que Horkheimer et Adorno firent remarquer que les autos, les bombes et les films apparemment sans rapport les uns avec les autres, assurent la cohésion du système (halten I...I das Ganze zusammen). Mais nos auteurs vont encore plus loin pour montrer que les interconnexions entre les divers objets techniques modifient le corps social dans son ensemble pour créer un gigantesque système à l'intérieur duquel la publicité apparaît comme un sous-système technique destinè à resserrer les liens qui lient les consommateurs aux grands trusts. Renchérissant, ils notent : " Même les oppositions politiques dans leurs manifestations esthétiques sont unanimes pour chanter les louanges du rythme d'airain de ce système. Des pays totalitaires aux autres pays, les bâtiments administratifs et les centres d'expositions industrielles se ressemblent presque tous par leur décoration $\gg{ }^{14}$. Nous nous trouvons en présence d'une rationalité uniformisante reposant sur l'abolition des différences et la négation de l'identité du Moi individuel : « l'identité de toutes les choses entre elles se paie par l'impossibilité de chaque chose d'être identique à elle-même $\gg^{15}$. Dilué dans la masse indifférenciée de la horde, le moi individuel se trouve ainsi conduit à sa perte par la rationalité

\footnotetext{
12. Horkheimer, M., Kritik zur instrumentellen Vemunft, op. cit., p. 31 -

13. Horkheimer / Adorno, Dialektik der Aufklärung, op. cit., p. I44.

14. Ibid., p. I44.

I5- Ibid., p. 23
} 
technique, rationalité de la domination par excellence. En prolongement à cette perspective, Marcuse développe une critique pénétrante de la société industrielle. Il montre en effet à quel point la production « idéologique » (''art, la poésie et le langage), en intériorisant les formes rationalisées de la domination au profit d'une intégration dans le système de production industrielle, se trouve dépouillée de son pouvoir de négation. Ainsi, l'art, la poésie et le langage qui subissent dans la société industrielle l'assaut de la rationalité technique ou rationalité du statu quo, perdent leurs éléments oppositionnels et transcendants, c'est-à-dire leur disposition à un authentique développement du sens. En défiant et mutilant le sens, la rationalité technique dans son expression et son expansion devient synonyme de thanatocratie (mort du négatif, anéantissement des forces oppositionnelles).

Cette situation a inspiré à l'École de Francfort un certain pessimisme sur l'avenir des sociétés industrielles avancées. Adorno, dans Minima Moralia souligne qu'« il ne peut avoir de vraie vie dans un monde qui ne l'est pas $»^{16}$. Horkheimer pour sa part affirme : « on ne pouvait dire que ce qui était mauvais dans la société actuelle $\gg 7$.

Mais cela n'empêcha nullement les philosophes francfortiens ${ }^{18}$ d'esquisser des éléments de solutions destinés à combattre le Mal social. On pourrait, non sans simplification excessive, subsumer les solutions de l'École sous les formules suivantes:

- surmonter (au sens fort de überwinden) la rationalité du statu quo par le développement des activités non techniques ou transtechniques;

- déterminer le champ d'exercice légitime de la rationalité technique et marquer sa lisière par la mise en route de ce que Habermas appelle rationalité herméneutique (Verständigungsorientierte Rationalität).

Ces deux formules jointes à la critique réitérée de la domination permirent aux philosophes francfortiens d'asseoir une théorie de la société industrielle avancée, théorie qui constitue à nos yeux une remarquable contribution à la philosophie de la technique.

I6. Adorno, Th. W., Minima Moralia, Frankfurt /M., Suhrkamp, 195I, p. 42.

17. Horkheimer, M., Gesellschaft im Übergang. Fischer, 1972, p. 164.

18. La différence entre le (philosophe) francfortien et le (philosophe) francfortois est qu'ils se situent respectivement dans des rapports d'appartenance « idéologique » et géographique. Ainsi par exemple, un philosophe originaire d'une ville africaine peut être francfortien s'il se réclame par sa production intellectuelle de l'Ecole de Francfort. 
Mais, si remarquable soit sa contribution, l'École de Francfort, à en croire les nouveaux philosophes de la technique, ne constitue pas une philosophie de la technique stricto sensu. Cela évidemment appelle une confrontation des positions de l'École avec celles des nouveaux philosophes de la technique.

\section{B. Éléments de comparaison : l'École de Francfort et les nouveaux philosophes de la technique}

Nous allons à présent procéder à la confrontation de deux tendances philosophiques dont l'une demeure jusque là inconnue. Peut-être aurait-il fallu procéder à une exposition préalable de chacune d'elles. Cette méthode qui ne manque certainement pas d'intérêt, présente néanmoins l'inconvénient de vouer le texte à des redites qui pourraient paraître rébarbatives. D'où le choix de l'orientation qui est ici la nôtre : dévoiler au fur et à mesure que se déploie la confrontation, les thèses fondamentales des nouveaux philosophes de la technique ${ }^{\mathrm{I} 9}$.

Toutefois, on pourrait en prélude à cette confrontation, s'interroger sur l'identité de ces philosophes en posant la question : qui sont les nouveaux philosophes de la technique? La réponse la plus élémentaire et en même temps la moins instructive est celle qui consiste à citer des noms. Plus édifiante nous paraît la démarche conduisant à identifier le principe fondateur et unificateur de leur contribution à la philosophie de la technique. Retenons pour l'essentiel que cette contribution d'inspiration pragmatiste est plus ou moins proche du technology assessment. En effet, affichant un anti-positivisme circonspect, ces philosophes partagent l'idée selon laquelle la dynamique technicienne interpelle aujourd'hui plus que jamais le philosophe; ils suggèrent que le philosophe réponde à cette interpellation par une évaluation de la technique solidaire d'une réévaluation de l'éthique. Ces indications très sommaires appellent évidemment une exposition détaillée des thèses propres à ces nouveaux philosophes. L'exposition de ces thèses se présentera sous le mode d'une confrontation laissant apparaitre clairement les analogies et les points de rupture entre les deux tendances philosophiques qui nous préoccupent.

\section{a) Analogies}

Il s'agit ici de «mettre sur le tapis » les points de convergence entre les tendances exprimées d'une part, par l'École de Francfort et d'autre

19. Les textes de ces philosophes étant pour la plupart seulement disponibles en allemand et en anglais, nous assumons, dans le cadre de la présente étude, l'entière responsabilité de leur traduction française. 
part, par la N.G.T. Le premier point de convergence entre ces deux tendances est la critique ou mieux la Technikkritik ${ }^{20}$. D'un côté comme de l'autre, s'opère une critique de l'aliénation techniciste, de la technocratie, et de la thèse du développement autonome du progrès technique (die These von der Eigengesetzlichkeit des technischen Fortschritts).

Il en découle ce second point de convergence qui est la prise en compte de la dimension sociale de la technique. Tous se refusent en effet à isoler la technique du collectif humain dans et par lequel naissent les objets techniques. Ce point de convergence peut être subsumé sous le concept de «Sozialphilosophie der Technik».

Un autre point de convergence est la méfiance à l'égard des spéculations métaphysiques oiseuses et de l'irrationalisme : tous sont animés du souci de proposer, sur la base de la prise en compte du monde vécu social (soziale Lebenswelt) et de la reconnaissance de la puissance technicienne, des solutions à la crise de la civilisation technicienne sans démordre de la raison.

Enfin, nous retiendrons comme dernier point de convergence, l'absence d'optimisme exorbitant à l'égard de la technique : loin de développer une quelconque « technophilie », ces auteurs ne donnent pas non plus dans la « technophobie ».

Toutefois, on ne saurait se fonder sur ces analogies pour prétendre connaître ou faire connaître les thèses propres à la NGT. Une analyse différenciée des positions de la NGT et de celles de l'École de Francfort s'en trouvera justifiée.

\section{b) Différences}

Les différences de tendances constatées entre l'École de Francfort et les nouveaux philosophes de la technique seront présentées de façon schématique et recensées sous le titre de décalage. Aussi convient-il de signaler que chaque décalage recensé fera l'objet d'une présentation autonome de façon à maintenir la liste continuellement ouverte.

\section{Décalage : l'objet de la critique}

La technique qui fait l'objet de la critique de l'École de Francfort semble correspondre au concept wébérien de rationalité téléologique (Zweckrationalität). C'est la technique prise dans un sens très élastique qui englobe presque toutes les techniques possibles ou mieux toutes les activités rationnelles orientées vers un but. Les nouveaux philosophes de la technique, mettant en garde contre ce concept

20. On peut dire que l'Ecole de Francfort en tant que « Technikkritik» préfigure la formation de la «neue Generation der Technikphilosophen ». 
générique (Gattungsbegriff) de technique et l'évaluation par trop généralisante qui en résulte, déclarent :

Ropohl : « Je plaide pour une critique différenciée de la technique $»^{21}$. Plus loin : «Plutôt que de condamner en bloc la technique ou de la louer pareillement, une critique différenciée de la technique doit permettre de dégager de façon réaliste et objective les véritables défaillances sectorielles de la présente technosphère $»^{22}$. Huning : « La nature de la technique ne se laisse pas cerner par une définition générale et abstraite $»^{23}$. Renchérissant, Friedrich Rapp brandit la Realtechnik (terme dû à von Gottl-Ottlilienfeld) comme objet privilégié de la philosophie de la technique.

Or, la Realtechnik, terme généralement traduit par technique du réel $^{24}$, ne constitue qu'une partie de la technique. C'est du moins ce que dénote ce concept dans la classification initiée par von GottlOttlilienfeld. On distingue en effet dans cette classification, en plus de la Realtechnik définie comme ensemble des pratiques axées autour de la maîtrise technique de la nature, la technique individuelle (Individualtechnik), la technique sociale (Sozialtechnik) et la technique intellectuelle (Intellektualtechnik). La technique individuelle est l'ensemble des « pratiques de transformation qui ont pour objet les réalités psychiques et corporelles de l'individu ». La technique sociale renferme « les pratiques de transformation qui ont pour objet les relations entre individus ». Ce sont entre autres les « techniques d'administration et d'éducation ». Quant à la technique intellectuelle, elle renvoie aux pratiques méthodologiques dont les objets relèvent du domaine intellectuel, comme celles qui permettent la résolution d'un problème ou d'une énigme, par exemple les techniques de calcul et de toute méthodologie en général ${ }^{25}$.

On peut donc reconnaître dans l'attachement à la Realtechnik l'indice d'un certain rèductionnisme. Mais la NGT serait-elle prête à assumer une telle déficience? Si non, comment expliquer leur intérêt pour la Realtechnik?

21. Ropohl, G., Die unvollkommene Technik, Frankfurt, Suhrkamp, 1985. P. 9.

22. Ibid., p. 85 .

23. Huning, A., « Der Technikbegriff » dans Technik und Philosophie. Encyclopédie, Tome I, Düsseldorf, Editions VDI, 1990, p. II.

24. Cf. J-Y. Goffi, La philosophie de la techmique, Paris, P.U.F. (Que sais-je ?), 1988, p. 23 .

25. Ropohl commentant von Gottl-Ottlilienfeld dans : Cahier STS, N 2, Paris, Editions du CNRS, 1988, p. 32. 
L'une des raisons alléguées par la NGT en faveur de la Realtechnik est qu'elle constitue à ses yeux le noyau dur (Kernpartie) de la technique, voire la technique par excellence. Tel fut également le parti pris par von Gottl-Ottlilienfeld.

Mais la NGT se sépare de von Gottl-Ottlilienfeld lorsqu'elle se résout à distendre le concept de Realtechnik (sans le rendre commensurable à la Zweckrationalität de Weber) en y intégrant les autres formes de technique par la mise en évidence formelle de l'artefact (Sachsystem). Ainsi, la NGT suggère que la Realtechnik ou Sachtechnik soit perçue là la fois et de façon complémentaire) comme : a) «ensemble des artefacts orientés vers des fins utiles 》; $b$ ) « ensemble des processus et dispositifs humains dans et par lesquels sont produits ces artefacts »; c) «ensemble des processus et pratiques humains dans et par lesquels sont utilisés ces artefacts $»^{26}$. C'est en ce sens qu'il faut comprendre la Realtechnik de la NGT comme objet privilégié de philosophie de la technique.

\section{Décalage 2: « thématisation » (Thematisierung) de la philosophie de la technique}

L'École de Francfort, il est vrai a esquissé certains thèmes majeurs de la philosophie de la technique; elle a incontestablement contribué au développement de cette nouvelle dimension de la philosophie qu'elle n'a malheureusement pas thématiseee. Nayant pas (volontairement ou involontairement ) thématisé la philosophie de la technique, l'École de Francfort fait aujourd'hui figure de philosophie implicite de la technique. Elle serait, selon l'expression de G. Ropohl une parathematische Technikphilosophie et ceci par opposition à la thematische Technikphilosophie. Tandis que la première expression sert à désigner toute philosophie abordant par des biais la question de la technique, la seconde quant à elle, dénote cette approche qui place la technique au coeur de la réflexion philosophique ${ }^{27}$. Ces différents concepts (parathematische et thematische Technikphilosophie) tracent pour ainsi dire la ligne de démarcation entre l'École de Francfort et les nouveaux philosophes de la technique.

\section{Décalage 3 : le modèle systémique (Systemmodell) ou la technique comme sous-système}

Concevoir la technique comme sous-système suppose soit l'adoption de l'approche systémique, soit l'intention explicite ${ }^{28}$ de dévoiler la nature systemique de la technique. Or, aucune de ces deux

26. Ropohl, G., Die unvollkommene Technik, Frankfurt, Suhrkamp, 1985, p. 6r.

27. Cf. Ropohl, G., Technologische A ufklärung, Frankfurt, Suhrkamp, I99I, p. II. 
hypothèses n'est applicable à la démarche des philosophes francfortiens et cela en raison de leur aversion pour le système.

C'est d'ailleurs cette aversion pour le système qui incita Adorno et Horkheimer à s'attaquer violemment au système hégélien qui, à leurs yeux, constituait le sommet de la systématicité. En effet, Hegel dont les efforts avaient consisté à réconcilier dans la Totalité du système le sujet et l'objet, la raison de la société civile et la raison d'Etat, fut accusé d'avoir hypostasié la réalité en la murant dans la Totalité abstraite, voire fausse : « das Ganze ist das Unwahre » ${ }^{29}$, le Tout (la totalité hégélienne) est le non-vrai. Récusant la Totalité hégélienne. Horkheimer a pu dire des analyses de Hegel qu'elles sont « des abstractions parfaitement dénuées de sens et nullement des âmes du réel $^{3^{\circ}}$. Pour Horkheimer, la Totalité hégélienne n'est rien d'autre qu'une raison instrumentale mystifiée et le système hégélien rien de plus qu'une rationalisation de la domination au lieu d'en être la critique. A l'appui de ces vues, Habermas entreprend une critique de l'approche systémique qui, selon lui, conduit à l'absorption de l'activité communicationnelle par des modèles de systèmes rigides et autorégulés.

L'École de Francfort de ce point de vue, est en parfait désaccord avec les nouveaux philosophes de la technique qui, au contraire manifestent un intérêt certain pour l'approche systémique. Mais dans l'esprit de ces philosophes, l'approche systémique ne doit ni donner lieu au déploiement de systèmes abstraits, antidialectiques et anthropocentrés ni céder le pas à la méthode cybernétique foncièrement liée à une intention technocratique.

C'est sans doute ce souci d'une prise en compte de l'humain et du technique dans une démarche systémique qui conduisit la N.G.T à forger le concept de système sociotechnique (soziotechnisches System) ${ }^{31}$. Ce concept, à en croire ces philosophes, permet de cerner de façon rigoureuse et systématique la dimension sociale de la technique (der gesellschaftliche Charakter der Technik) ainsi que la dimension technique de la société (der technische Charakter der Gesellschaft).

28. Nous disons bien intention explicite. Cette précision est importante; car la description de la technique comme sous-système est bel et bien présente dans le discours de l'Ecole de Francfort. Toutefois, ce discours ne procéde pas à une mise en évidence formelle de cette dimension de la technique.

29. Adomo, Th. W., Drei Studien zu Hegel, Frankfurt, Suhrkamp, 1963. p. 104.

30. Horkheimer, M., Anfänge der bürgerlichen Geschichtsphilosophie. Hegel und das Problem der Metaphysik, Frankfurt, Fischer, 1970. p. go.

31. G. Ropohl, le promoteur de ce concept, nous en donne la représentation graphique que voici : 


\section{Décalage 4 : la rếérence à l'individu}

La référence à l'individu est excessive du côté de l'École de Francfort. En effet, à l'exception de Habermas (cofondateur de la Makroethik caractérisée par la transindividualité), les membres de l'École prirent constamment parti pour l'individu, mieux pour la particularité contre la totalité fausse. «Das Ganze ist das Unwahre » disait Adorno. D'où l'invocation chez Adorno, de l'art comme moyen d'èmancipation de l' individu. Mais si l'art offre de telles possibilités, c'est d'abord parce qu'il permet de sauver le particulier (das Besondere zu erretten) puisque la particularité lui est immanente.

Tandis que l'École de Francfort privilégie la catégorie individuelle, les nouveaux philosophes de la technique quant à eux, s'attèlent à montrer le caractère inopérant du particularisme face à la crise actuelle de la civilisation technicienne et surtout à la problématique de l'agir (éthique) dans les sociétés contemporaines. Contre l'orientation particulariste / individualiste de l'éthique, l'argumentation des nouveaux philosophes de la technique est schématiquement la suivante:

« Le developpement technique s'est arraché à la sphère individuelle pour devenir une fonction de systèmes sociotechniques complexes. Or ce qui se déploie suivant les lois d'un système, ne peut plus être justifié

$3 \mathbf{I}$

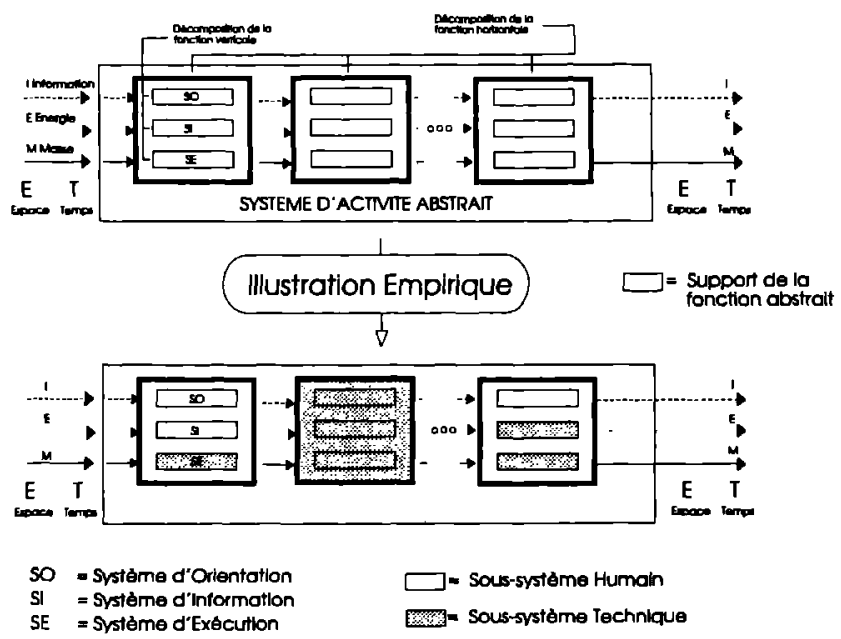

UN MODELE DE SYSTEME SOCIO-TECHNIQUE ABSTRAIT ET CONCRET Notre tradurtion et edaptation.

32. Ropohl, G., « Ob man die Ambivalenzen des technischen Fortschritts mit einer 
à l'échelle individuelle > $^{32}$. Il appert « que les appels moraux adressés aux individus sont nécessairement vains quand on sait que les conséquences des actions morales sont avant tout imputables non au sujet individuel mais au système complexe émanant de l'action collective ` $^{33}$. Ces présupposés, estiment les nouveaux philosophes de la technique, doivent être le support de toute réflexion éthique soucieuse de gagner en autorité auprès de nos contemporains. Attentifs aux sollicitations des contemporains, et forts des présupposés susindiqués, ces philosophes peuvent se targuer d'intervenir valablement dans le processus d'évaluation de la technique (Technikbewertung ou Technikfolgenabschätzung) devenu leur « champ réflexif » par excellence.

\section{Pour conclure}

La philosophie de la technique, comme toute discipline naissante, a connu un « long hiver » avant de revêtir la forme qui est aujourd'hui la sienne.

Apparue d'abord sous la forme d'un anthropologisme instrumentaliste (Ernst Kapp), ensuite comme une métaphysique néoplatonicienne de la technique (Dessauer) et enfin comme une ontologie de la technique (Heidegger), la philosophie de la technique « achève » son parcours sur le topos de l'évaluation de la technique (Technikbewertung). Dans la Technikbewertung s'accomplit ce double mouvement dialectique : la spiritualisation de la technique ou dialectique ascendante d'une part, et d'autre part, la matérialisation de la réflexion philosophique ou dialectique descendante. Dans ce mouvement dialectique, la contribution de l'École de Francfort fut certes indéniable mais partielle. Elle a en effet laissé à mi-chemin le versant descendant de cette dialectique que les nouveaux philosophes de la technique tentent non sans difficulté de pousser à son achèvement. C'est en fait avec l'entrée en scène philosophique de la N.G.T que la philosophie de la technique a connu en Allemagne son véritable essor.

Mais l'essor de la philosophie de la technique en Allemagne nous paraît liéa deux facteurs essentiels qui trouvent dans la conscience leur point d'ancrage.

Il y a d'abord la conscience aiguë des problèmes liès à la dégradation de leur environnement. Ce à quoi il faut ajouter le développement dans la conscience des intellectuels allemands de la

neuen Ethik meistem kann ? », dans Technikverantwortung, hg. v. H. Lenk et M. Maring, Frankfurt / New York, Campus, 199I. p. 57. . 
conviction selon laquelle la dynamique technicienne appelle d'innombrables facteurs transcendant les compétences de l'ingénieur ou du technicien. Cette conviction est exprimée en ces termes : «Die Technik ist heute und künftig zu wichtig, als daß man sie allein den Technikem überlassen dürtte »34. Loin d'être un simple flatus vocis, cette idée se trouve éminemment traduite dans les faits et cela de façon multiforme. Deux faits majeurs retiennent notre attention.

Il s'agit d'une part, de la relation singulièrement étroite qui s'est établie entre la NGT et la VDI (Verein Deutscher Ingenieure = Association des Ingénieurs Allemands). C'est ici le lieu de signaler qu'il n'est pas rare de trouver en Allemagne des travaux co-signés par des philosophes et des ingénieurs ou des travaux de philosophes édités avec le concours de la VDI.

On retiendra d'autre part, la présence du philosophe à l'entreprise. En tant que penseurs de la relation èthico-technique, les nouveaux philosophes allemands parviennent à substantialiser leur projet de prise en compte de la technique en intervenant de façon décisive au niveau de ce qu'on appelle «Ethik der Personalführung » : entendez simplement gestion des ressources humaines avec ce que cela comporte comme implications éthiques et / ou transtechniques. Ces philosophes peuvent ainsi se targuer de contribuer in concreto à l'action collective de maîtrise du phẻnomène technique, un phénomène qui ne peut être véritablement maîtrisé que par la prise de conscience de son caractère transinstrumental ainsi que par la mise en route d'activités visant à préserver l'essence axiologique de l'homme vivant dans le technocosme.

Nous arrêtons ici cette petite exploration du paysage germanique de la philosophie de la technique en souhaitant vivement que notre « regard » en croise bien d'autres qui, à n'en pas douter, viendront enrichir cette étude qui constitue à nos yeux une contribution assez modeste à la (re)construction d'une histoire de la philosophie de la technique.

Université Nationale de Côte-d'Ivoire

34. Nous traduisons : « La technique est pour les temps présents et à venir (une réalité) trop importante pour qu'on se permette de la laisser aux mains des seuls techniciens $\gg$. 


\section{BBIIOGRPPHIS SiltCIIIIE}

Tous les ouvrages mentionnés dans le corps du texte ne sont pas repris dans cette bibliographie.

ADORNO, Th. W., Minima Moralia, Frankfurt, Suhrkamp, 195ı.

Noten zur Literatur, Frankfurt, Suhrkamp, 1958.

Negative Dialektik, Frankfurt, Suhrkamp, 1966.

Ästhetische Theorie, Euvres complètes, vol. 7, 1970.

DESSAUER, F., Philosophie der Technik. Das Problem der Realisienung, Bonn, Cohen, 1927.

HABERMAS, J., Technik und Wissenschaft als Ideologie, Frankfurt, Suhrkamp. 1968.

«Technischer Fortschritt und sociale Lebenswelt 》 dans H. Kreuzer (Hrsg) : Literarische und naturwissenschaftlische Intelligenz. Dialog über die « zwei Kulturen », Stuttgart, 1969 .

HORKHEIMER, M. et ADORNO, Th., Dialektik der Aufklärung, Amsterdam, Querido, 1955.

HORKHEIMER, M., Kritik zur instrumentellen Vernunft, Frankfurt, Fischer, 1967. Anfänge der bürgerlichen Geschichtsphilosophie, Hegel und das Problem der Metaphysik, Frankfurt, Fischer, 1970.

Gesellschaft im Übergang, Frankfurt, Fischer, 1972.

KAPP, E., Grundlinien einer Philosophie der Technik, Braunschweig, Druck und Verlag v. G. Westermann, 1877.

LENK et MOSER, Techne, Technik Technologie, München, Uni-Taschenbuch, 1973.

LENK et ROPOHL (hrg.), Ethik und Technik, Stuttgart, Reclam, 1987.

MARCUSE, H., Kultur und Gesellschaft, I / II, Frankfurt, 1965.

L'homme unidimensionnel, traduction M. Wittig, Paris, Minuit, 1968.

RAPP, F., Analytische Technikphilosophie, Freiburg, München, Karl Albert, 1978. Technik und Philosophie, Ouvrage collectif / Encyclopédie, VDI, Düsseldorf, I990.

ROPOHL, G., Eine Systemtheorie der Technik, München / Wien, Hanser, 1979.

Die unvollkommene Technik, Frankfurt, Suhrkamp, 1985.

Technologische Aufklärung. Beiträge zur Technikphilosophie, Frankfurt, Suhrkamp, 1991. 